\title{
FMH Factoring Services - damit Sie sich auf Ihre Medizin konzentrieren können
}

\author{
Max Giger ${ }^{a}$, Reinhard Kunz ${ }^{b}$ \\ a Präsident FMH Services \\ b Geschäftsführer FMH Services
}

Korrespondenz: Reinhard Kunz FMH Services Burghöhe 1 CH-6208 Oberkirch
Wir freuen uns, Ihnen unsere neue Dienstleistung - FMH Factoring Services - vorzustellen.

Mit FMH Factoring Services erweitern wir die heutigen elektronischen Honorarabrechnungssysteme für freipraktizierende Ärzte und Ärztinnen mit einem echten Online-Factoring. Gemeinsam mit der mediserv AG in Biel, einem seit Jahren erfolgreichen Spezialisten dieser Dienstleistung, bieten wir der Ärzteschaft die effizienteste Fakturierungsform. Mit FMH Factoring Services übernehmen wir Ihre gesamten Fakturierungs-, Mahn- und Inkassoaktivitäten. Sie verfügen sofort über das Geld und können sich auf Ihre ärztlichen Leistungen konzentrieren. Dank einer elektronischen Schnittstelle erfolgt der gesamte Datenaustausch mit uns per Mausklick.

Das echte Factoring entlastet Sie von allen Aktivitäten aus der Rechnungsstellung und dem Debitorenrisiko. Dank der Online-Anbindung und eines automatischen Datentransfers verfügen Sie umgehend über die fakturierten Beträge auf Ihrem Kundenkonto. Der Kaufpreis von mediserv AG für Ihre Forderung entspricht dem Rechnungsbetrag abzüglich einer geringen Gebühr. Ihre Einsparungen sind erheblich: Kontokorrentkredite, Kosten der Fakturierung mit Versand, Inkassokosten und Arbeitsaufwand wegen Zahlungsproblemen gehören so der Vergangenheit an. Lassen Sie Ihre Guthaben auf dem mediserv-Konto stehen, so werden diese mit $2 \%$ pro Jahr verzinst.

Voraussetzung für eine Zusammenarbeit ist der Einsatz einer Software zur Praxisadministration mit eigener Fakturierung. Gemeinsam mit den führenden Herstellern von Schweizer Praxissoftwareanbietern haben wir geeignete Schnittstellen entwickelt.

Daraus ergeben sich gewichtige Vorteile:

- nahezu 100\%ige Entlastung im Debitorenmanagement, im Druck und Versand der Patientenrechnungen und im Mahnwesen;

- Wegfall der Refinanzierung durch die Bank dank einer sofortigen Verfügbarkeit ohne Wartezeit;

- keine Debitorenverluste im Factoring;

- Zeit- und Energiegewinn für Sie;

- Einsatz modernster Technologie und Erfüllung aller Sicherheitsstandards und Datenschutzvorschriften;

- neuartige, online festlegbare Zahlungspläne für Ihre Patientinnen und Patienten, wie Ratenzahlung bis 24 Monate (bis vier Raten ohne Mehrkosten);

- individuelle Mahnfristen oder die Angabe von Absenzen im Abwesenheitsassistenten sind online in wenigen Schritten durchführbar;

- Einsatz unseres patientengerechten, bewährten Inkassoverfahrens durch FMH Inkasso Services.

Die EDV-gestützte Administration ist aus dem heutigen Praxisalltag nicht mehr wegzudenken. Die wachsenden administrativen Aufgaben, u. a. Anfragen von Versicherern und Dokumentationspflichten, bedingen bereits einen erheblichen Zeitaufwand. Mit FMH Factoring Services wollen wir diesen verringern und Ihnen eine neue Möglichkeit bieten, die gesamten Abrechnungsverfahren auszulagern und Ihre Zahlungssysteme unabhängig von Banken und Betriebskrediten zu gestalten.

Dank dieses im nördlichen Nachbarland bereits von Tausenden Ärztinnen und Ärzten geschätzten und erfolgreich angewandten Onlineverfahrens können Sie sich auf die Betreuung Ihrer Patientinnen und Patienten konzentrieren. Wir übernehmen für Sie die Abwicklung der Zahlungs-, Mahn- und Inkassoprozesse. Dank des integrierten Factorings verfügen Sie sofort über die Geldforderungen, und gleichzeitig erzielen Sie Einsparungen, die die Factoringgebühr mehr als refinanzieren.

Wir freuen uns, Sie zu beraten. 